\title{
Drugs and precursor sensing by complementing low cost multiple techniques: overview of the European FP7 project CUSTOM
}

\author{
Alberto Secchi*a ${ }^{\mathrm{a}}$, Anna Maria Fiorello ${ }^{\mathrm{a}}$, Massimiliano Dispenza ${ }^{\mathrm{a}}$, Sabato D'Auria ${ }^{\mathrm{b}}$, Antonio \\ Varriale $^{\mathrm{b}}$, Alessandro Ulrici ${ }^{\mathrm{c}}$, Renato Seeber ${ }^{\mathrm{c}}$; Juho Uotila ${ }^{\mathrm{d}}$; Vincenzo Venditto ${ }^{\mathrm{e}}$, Paolo Ciambelli ${ }^{\mathrm{e}}$, \\ Juan Carlos Antolín ${ }^{\mathrm{f}}$; Francesco Colao ${ }^{\mathrm{g}}$, Tom Kuusela ${ }^{\mathrm{h}}$, Ilkka Tittonen ${ }^{\mathrm{i}}$, Päivi Sieviläi, Gregory \\ Maisons ${ }^{1}$ \\ ${ }^{a}$ SELEX Sistemi Integrati, Via Tiburtina, 00131 Rome, Italy; ${ }^{b}$ Consiglio Nazionale delle Ricerche, \\ Via Pietro Castellino, 80131 Napoli, Italy; ${ }^{\mathrm{C}}$ Università di Modena e Reggio Emilia, Italy; ${ }^{\mathrm{d}}$ Gasera \\ Ltd., Finland; ${ }^{\mathrm{e}}$ Università di Salerno, Italy, ${ }^{\mathrm{f}}$ TECNALIA Research and Innovation, Mikeletegi 2, San \\ Sebastian, Spain; ${ }^{\mathrm{g}}$ ENEA, Italy; ${ }^{\mathrm{h}}$ University of Turku, Finland; ${ }^{\mathrm{i}}$ Aalto University, Finland, ${ }^{1}$ 3-5 LAB, \\ France
}

\begin{abstract}
A large number of techniques for drug precursors chemical sensing has been developed in the latest decades. These techniques are able to screen and identify specific molecules even at very low concentration in lab environment, nevertheless the objective to build up a system which proves to be easy to use, compact, able to provide screening over a large number of compounds and discriminate them with low false alarm rate (FA) and high probability of detection (POD) is still an open issue. The project CUSTOM, funded by the European Commission within the FP7, deals with stand alone portable sensing apparatus based on multiple techniques, integrated in a complex system with a complimentary approach. The objective of the project is to achieve an optimum trade-off between opposite requirements: compactness, simplicity, low cost, sensitivity, low false alarm rate and selectivity. The final goal is the realization of an optical sensing platform able to detect traces of drug precursors compounds, such as ephedrine, safrole, acetic anhydride and the Benzyl Methyl Ketone (BMK). This is reached by implementing two main sensing techniques: the fluorescence enhanced by the use of specially developed Organic macro-molecules, and a spectroscopic technique in Mid-IR optical range.

The fluorescence is highly selective with respect to the target compounds, because it is based on properly engineered fluorescent proteins which are able to bind the target analytes, as it happens in an 'immune-type' reaction. The spectroscopic technique is based on the Photo-Acoustic effect, enhanced by the use of a widely Tunable Quantum Cascade Laser.

Finally, the sensing platform is equipped with an air sampling system including a pre-concentrator module based on a sorption desorption cycles of a syndiotactic polystyrene polymer.
\end{abstract}

Keywords: Drugs Precursor, LASER Photo Acoustic Spectroscopy, Biochemical Fluorescence, Preconcentrator, Syndiotactic Polystyrene, Mid Infra Red Quantum Cascade LASER, Cantilever.

\section{INTRODUCTION}

"Drug Precursors" ${ }^{[1]}$ are chemical substances having wide licit uses (e.g. in the manufacture of pharmaceutical products, perfumes, cosmetics, fertilizers, oils, etc.). However, they can be extremely dangerous when diverted from the licit channels for the illicit manufacture of drugs (such as heroin, cocaine, ecstasy, amphetamines, etc.).

For example 200g of piperonal (a substance commonly used in perfumery, in cherry and vanilla flavourings, in organic synthesis, and in the manufacture of mosquito repellents) are enough to produce 4,000 street doses of ecstasy.

*asecchi@selex-si.com; phone 0039 (0)6 4150 3712; fax 0039 (0)6 4150 3701; www.selex-si.com 


\begin{tabular}{cc}
\hline TABLE I & TABLE II \\
\hline Acetic anhydride & \\
N-Acetylanthanilic acid & \\
Ephedrine & Acetone \\
Ergometrine & Anthranilic acid \\
Ergotamine & Ethyl ether \\
Isosafrole & Hydrochloric acid \\
Lysergic acid & Methyl ethyl ketone \\
3,4-Methylenedioxyphenyl-2- & Phenylaceticacid \\
propanone & Piperidine \\
Norephedrine & Sulphuric acid \\
1-Phenyl-2-propanone & Toluene \\
Piperonal & \\
Potassium permanganate & \\
Pseudoephedrine & \\
Safrole &
\end{tabular}

Table 1 - Tables I and II of the United Nations Convention, 1988.

The list of the drug precursors chemicals according to the article 12 of the 1988 United Nations Convention against the Illicit Traffic in Narcotic Drugs and Psychotropic Substances ${ }^{[2]}$ is reported in table 1. The listed 23 substances are referred as essentials (Table I), if they are used to refine and process plant-based drugs chemicals and can be readily replaced by other chemicals with similar properties, or as precursors (Table II), if they are used as starting chemicals inputs for the production of synthetic drugs and are less likely to be substituted.

The need of a portable sensing platform able to perform chemical detection and identifications over a large number of compounds is a fundamental requirement of the law enforcement agencies to control the manufacture and the distribution of illegal narcotics and synthetic substances worldwide.

We present in the following the most recent results in the development of the sensor for drug precursors within the EU funded project CUSTOM ${ }^{[3]}$ ("Drugs and Precursors Sensing by Complementing Low Cost Multiple Techniques", FP7SEC-2009.1.3-028). It focuses on the point detection methodologies due to its applicability in custom, airport and harbor check point scenarios, where inspection of trucks, cars, containers, as well as people and baggage is required.

\section{TECHNOLOGY DEVELOPMENT}

A large number of different non-invasive hand held chemical sensors available in the market have been developed in the last decades. All of them are mainly based on a single detection approach, whether Ion Mobility Spectrometry (IMS), Surface Acoustic Wave (SAW), Gas Chromatography or Raman Spectroscopy.

In order to achieve an optimum trade-off between opposite requirements in terms of high probability of detection and low false alarm rate, the sensor architecture has been conceived to integrate a multiple sensing techniques, which operate in a complimentary approach: the Biochemical Fluorescence (BF) and the Laser Photo Acoustic Spectroscopy. A further improvement of the sensor selectivity is achieved by implementing a chemical Pre-Concentrator, based on the sorption/desorption cycles of a Syndiotactic Polystyrene (SP) polymer.

Starting from the functional architecture of the sensor, this chapter aims to present and discuss the current status of development of the main modules of the sensor.

\subsection{Architecture of the sensor}

The general concept of the sensor architecture is schematically showed in Figure 1. A micro-pump delivers the air samples to the Laser Photo Acoustic Spectrometer (LPAS) or to the Biochemical Fluorescence (BF) sensor.

Depending on the configuration of the bistatic valves and the path of the hydraulic circuit the three main functions can be accomplished. They are the LPAS and the BF loading, either directly or through the SP-PC, and the cleaning procedure of the modules. 


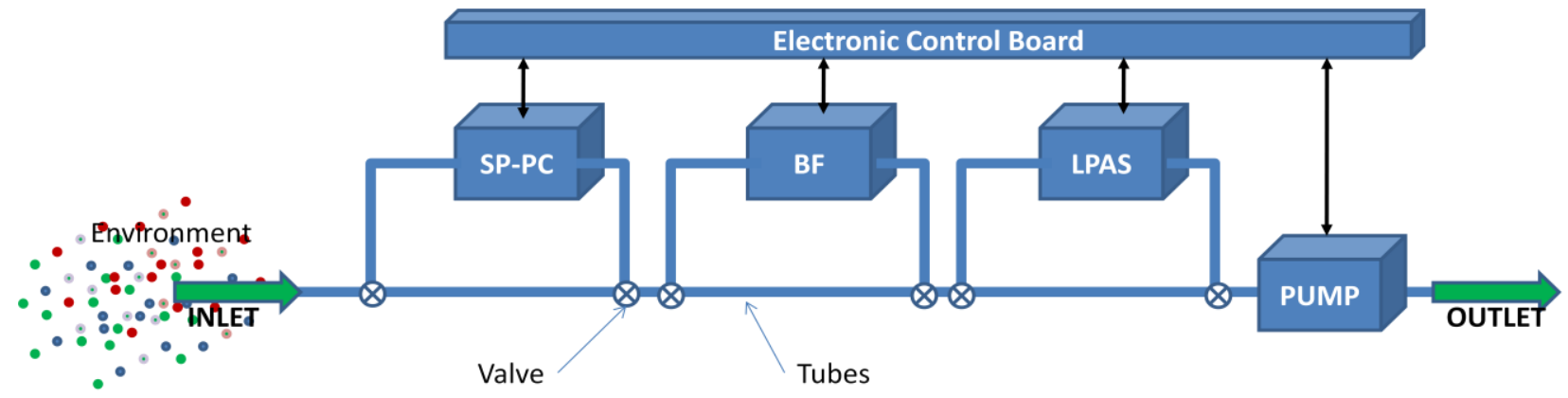

Figure 1: Schematic view of the sensor architecture and hydraulic connections between the Syndiotactic Polystyrene Preconcentrator (SP-SPC), the Biochemical Fluorescence (BF) module and the Laser Photo Acoustic Spectrometer (LPAS).

The sensor has two main operational modes:

1) A high Probability of Detection (PoD), where the LPAS module is used for a fast screening of the gas samples,

2) A low False Alarm (FA) rate, where the BF module is used to confirm the presence of target analyte.

The two operational modes are complimentary and are used in sequence. As the LPAS is generally faster than the BF, it is used in the "high POD" mode to make a rapid check of the presence of the target analyte at first. Whenever the probability of the target molecule presence is above a fixed threshold, the operative mode is changed to the more selective and accurate low FA rate mode, which makes use of the BF, to verify the detection.

Depending on the field of application, the SP-PC could be used to increase the sensitivity of the sensor, or bypassed to reduce the air sapling time and the power consuming.

An electronic control board is deputed to manage all the hardware modules of the sensor (SP-PC, BF, LPAS), as well as the data processing to elaborate the spectra acquired by the LPAS, the communication functions between each modules of the sensor, the user interfaces and the power supply.

\subsection{Laser Photoacoustic spectrometer}

Among various detection schemes, the LASER Photo Acoustic Spectroscopy (LPAS) offers some unique features such as low sample volume, high sensitivity and wide linear dynamic range. Signal is generated only from the absorption thus being a so called zero-background technique ${ }^{[4]}$.

The initial prototype of the LPAS sensor (see picture in figure 2) is a $320 \mathrm{~mm}$ x $141 \mathrm{~mm} \times 184 \mathrm{~mm}$ module, which consists on the quantum cascade laser, the photoacoustic cell and the readout interferometer. It is enclosed in a vibration isolated baseplate which integrates also the electronics and the air cooling.

The laser used for the initial prototype is a widely tunable External Cavity Quantum Cascade Laser (EC-QCL) delivering $15 \mathrm{~mW}$ of output power in the MID-IR (1200 cm-1) range with $100 \mathrm{~cm}-1$ of wavenumber tunability. The final EC-QCL prototype will have $200 \mathrm{~mW}$ power and $200 \mathrm{~cm}-1$.

In order to establish the presence of the target analyte, the acquired spectra coming from the LPAS module are processed and elaborated using the Partial Least Squares - Discriminant Analysis (PLS-DA) method. For a given set of wavenumbers, this multivariate method calculates one model for each drug precursor, giving its probability of presence. The advantages offered by this multivariate method consist in the fact that it does not only consider the useful (discriminant) information brought by each variable (wavenumber) separately, but also the information deriving from the interactions among all the analyzed variables.

In order to select the optimal wavelengths range to efficiently detect the drug precursor molecules in presence of interfering species and background air, a complex strategy of spectral response simulation has been developed ${ }^{[5]}$. For this purpose spectra of gases from literature databases have been collected, denoised by means of the Wavelet Transform and mixed together according to a concentration matrix. The optimal range has been defined by maximizing the classification efficiency for all the target gases by means of PLS-DA. 

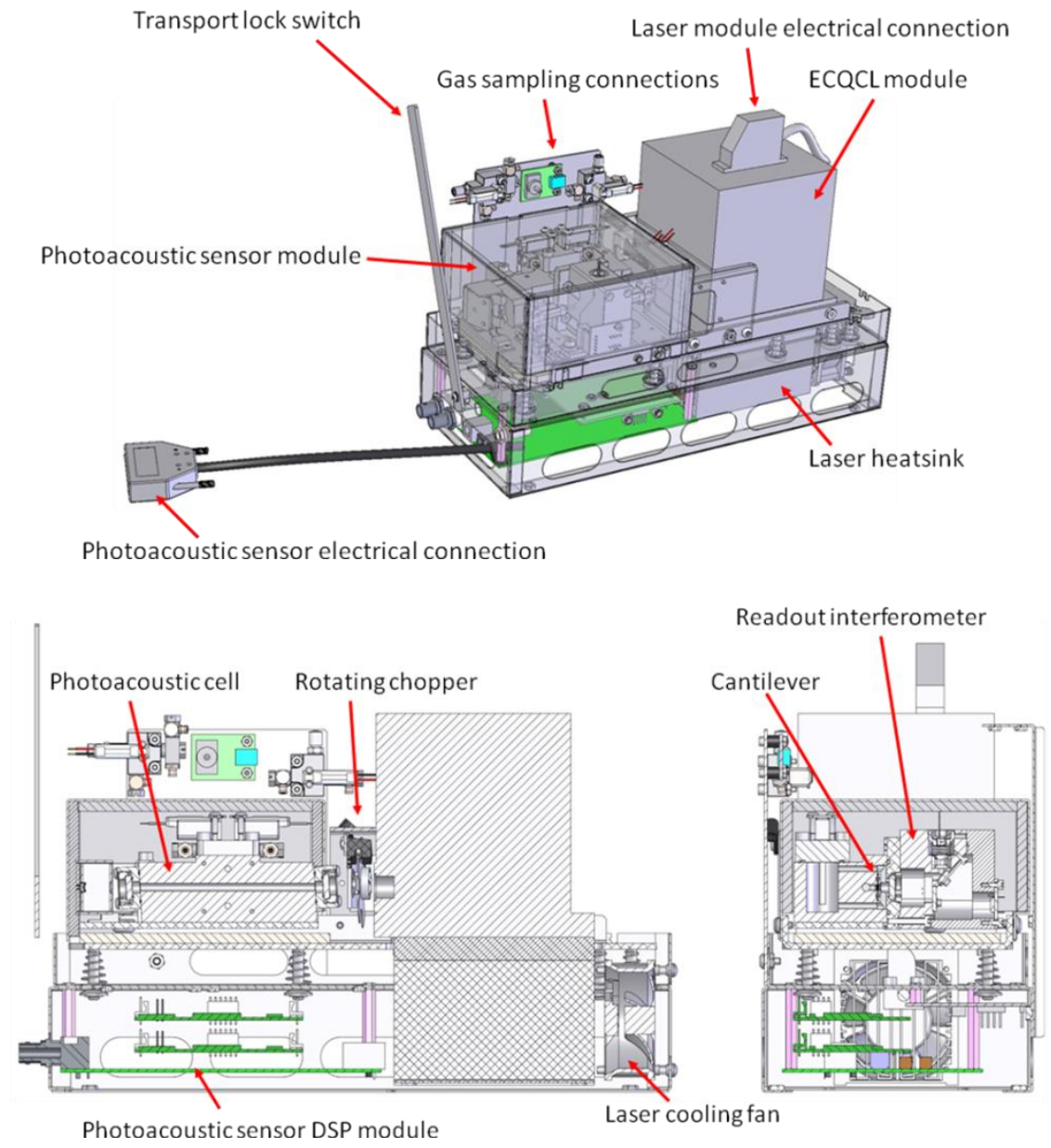

Figure 2 - Layout of the LPAS module. It consists on the quantum cascade laser, the photoacoustic cell and the readout interferometer. It is enclosed in a vibration isolated baseplate which integrates also the electronics and the air cooling. The whole dimensions are a $320 \mathrm{~mm} \times 141 \mathrm{~mm} \times 184 \mathrm{~mm}$.

The cantilever-type pressure sensor is designed to improve the sensitivity of PAS ${ }^{[6]}$. It is made out of single crystal SOIsilicon with a specially developed dry-etching process that leads to a highly stable component; this is why the sensor is practically totally immune to temperature and humidity variations. In addition, the sensor is not suffering from wearing.

The displacement of the cantilever is measured by a spatial type optical interferometer. It avoids the so called "breathing effect of the pressure sensor readout, which occurs in capacitive measurement principle, where the other electrode damps the movement of the sensor.

The EC-QCL is located on the same vibration damped baseplate as the photoacoustic cell, which is a $95 \mathrm{~mm}$ long thermally stabilized gold coated cylinder having $4 \mathrm{~mm}$ diameter.

The beam is chopped by a tuning fork located in front of the photoacoustic cell. A thermopile is used for sampling the laser power.

\subsection{Biochemical Fluorescence based Module}

The first prototype of the fluorescent module is based on a competitive immunoassay ${ }^{[7]}$. It is combined with an immunochemical approach to bind the target analyte, which is detected as a variation of fluorescence intensity by means of the Förster Resonance Energy Transfer (FRET) method. 
Förster resonance energy transfer (FRET) is a mechanism that relies on the energy transfer between two fluorophores. A donor fluorophore, initially in its electronic excited state, may transfer energy to an acceptor fluorophore (in proximity, typically less than $10 \mathrm{~nm}$ ) through non radiative dipole-dipole coupling.

The target compound contained in the sample competes to bind a specific antibody with a derivative of the target compound.

For their importance in the drug precursors illegal trafficking, the target analytes first considered for the preliminary experiments were the 1-Phenyl-2-propanone (BMK) and the Ephedrine.

One of the main tasks for the realization of the fluorescence sensor is the production of the reagents for the detection of the target compounds, namely anti ephedrine and anti BMK antibodies and ephedrine and BMK and their derivatives.

The Biochemical Fluorescent (BF) based module hardware (see prototype picture in figure 3) consists of an analysis module, the sample interface and a reagents handling module which comprises the fluidic system and some printed circuit boards together with the local control board. This control board contains a tiny microcontroller to acquire some process signals like temperature and reservoirs pressure. Besides, it controls the activation of the valves and the peristaltic pump in order to inject the required compounds depending on the sample target to be detected. Moreover, it enables the light source to excite the optochip and it receives the emitted signal of the sample after suitable conversions.

Providing the request of the Main Control Board (MCB) to detect some specific target, the local controller fills up a $450 \mu \mathrm{l}$ cuvette with the suitable quantity of required biomolecules and reagents. Afterwards, the local tiny microcontroller requests to the MCB the activation of the air pump to bubble the sample target passing through the preconcentrator if it were needed. After the mixture fills up the measuring cell, the local microcontroller switches on the light source exciting the optochip with a specific wavelength depending on the sample target. At the same time the detector, a Photo Multiplier Tube, will capture the specific wavelength light emitted by the optochip blocking other wavelengths by means of a band pass filter. After measuring, the fluidic system is cleaned to get ready for a new measurement.

In the case of the BMK detection, BMK antibody labeled with CF568 dye is excited with a $100 \mathrm{~Hz}$ pulsed light source of $535 \mathrm{~nm}$ and the emission from the sample is blocked with a band pass filter of $671 \mathrm{~nm}$.

The acquired raw data and some calculated mean values of the received light as voltage values are sent by the local microcontroller to the MCB through a Universal Asynchronous Receiver/Transmitter (UART) controller. The mean value is introduced into a calibrated curve in order to determine the concentration of the target.

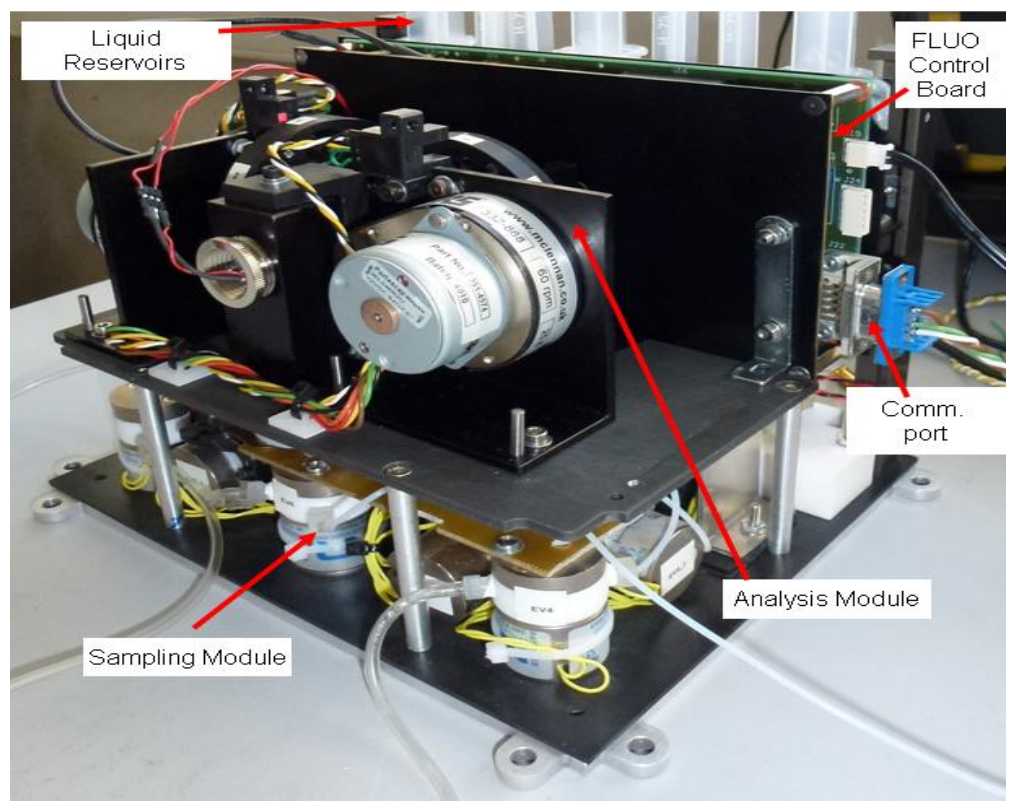

Figure 3 - Layout of the BF module. It consists of an analysis module, a handling module to manage liquids and the electronic boards which control and monitor its status 


\subsection{Preconcentrator}

The Pre-Concentrator (PC) is based on a nanoporous polymer material: the syndiotactic polystyrene (sPS), which is in form of aerogels beads with features of 500-1000 microns. It is used for its high sorption capacity and fast sorption/desorption kinetics with Volatile Organic Compounds (VOCs) ${ }^{[8]}$.

The preconcentrator consists on a metallic serpentine path of $500 \mathrm{~mm}$ length and of $2 \mathrm{~cm} 3$ of volume (see layout in figure 4) filled with about $300 \mathrm{mg}$ of sPs. It has $90 \%$ porosity and so very low density: of about $0.1 \mathrm{~g} / \mathrm{cm} 3$.

If thermal cycles are applied upon the PC, sPS will adsorb and concentrate VOC at low temperature $\left(-10^{\circ} \mathrm{C}\right)$, during air sampling, and it releases them at higher temperature (e.g. $\left.+40^{\circ} \mathrm{C}\right)$.

Preliminary experiments conducted on the PC unit by using acetic anhydride, demonstrate an improvement of a factor 13 of the concentration (from 5ppm to 65ppm) of the analyte in the sampled air. The use of sPS based pre-concentrator hence improves sensitivity of whole sensors as well as its detection limit and selectivity.

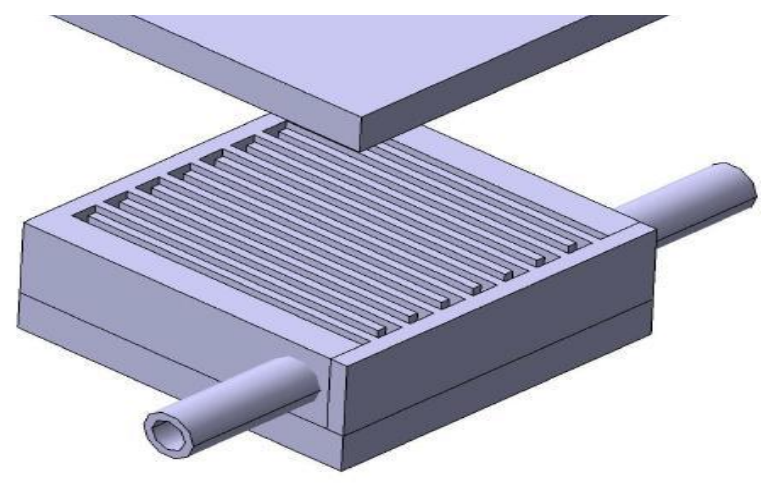

\section{CONCLUSION}

Figure 4 - Preconcentrator layout

In this paper we showed the work done up to now in the development of the novel sensor for drugs and drugs precursor. The features and the preliminary characterizations of the key components of the sensor: the QCL-PAS, the fluorescence module and the chemical pre-concentrator unit have been presented as self standing devices.

In the remaining part of the project, after final integration of the sensor, more detailed tests will be conducted in laboratory and real environment.

\section{REFERENCES}

[1] http://ec.europa.eu/taxation_customs/customs/customs_controls/drugs_precursors/index en.htm

[2] http://www.incb.org/incb/en/convention_1988.html

[3] www.custom-project.eu

[4] J. Uotila, J. Raittila, I. Kauppinen and J. Kauppinen, "Sensitive analysis of trace gases by using cantilever enhanced photoacoustic cell combined with a quantum cascade laser" 6th International Conference on Advanced Vibrational Spectroscopy (ICAVS6), June 2011.

[5] Ulrici, Calderisi, R. Seeber, J. Uotila, A. Secchi, A. Fiorello, M. Dispenza, "A feature selection strategy for the development of a new drug sensing system", Convegno nazionale sensori Proceedings, Rome, 2012

[6] 11. P. Sievilä, N. Chekurov, J. Raittila and I. Tittonen, "Sensitivity-improved silicon cantilever microphone for acousto-optical detection", submitted and under review for publication in Sensors and Actuators A: Physical, January 2012

[7] A.Varriale, S. D'Auria, “An immuno-based surface plasmon resonance biosensor for ephedrine detection”, Sec.+ Def. Conf.Prague 19 -22 Sept (2011), SPIE paper 8189A-21

[8] Vincenzo Venditto, "Nanoporous crystalline polymers with sulfonated amorphous phases: highly efficient vocs sorbent materials", XII Congresso Nazionale di Chimica dell'Ambiente e dei Beni Culturali 